\title{
Enhancement of local pairing correlations in periodically driven Mott insulators
}

\author{
Francesco Peronaci, ${ }^{1,2,3, *}$ Olivier Parcollet, ${ }^{1,4}$ and Marco Schiró ${ }^{5, \dagger}$ \\ ${ }^{1}$ Université Paris-Saclay, CNRS, CEA, Institut de Physique Théorique, 91191 Gif-sur-Yvette, France \\ ${ }^{2}$ CPHT, Ecole Polytechnique, CNRS, Université Paris-Saclay, 91128 Palaiseau, France \\ ${ }^{3}$ Max Planck Institute for the Physics of Complex Systems, Dresden 01187, Germany \\ ${ }^{4}$ Center for Computational Quantum Physics, Flatiron Institute, 162 Fifth Avenue, New York, New York 10010, USA \\ ${ }^{5}$ JEIP, USR 3573 CNRS, Collège de France, PSL Research University, 11 place Marcelin Berthelot, 75231 Paris Cedex 05, France
}

(Received 1 April 2019; revised manuscript received 27 January 2020; accepted 9 March 2020; published 1 April 2020)

\begin{abstract}
We investigate a model for a Mott insulator in the presence of a time-periodic modulated interaction and a coupling to a thermal reservoir. The combination of drive and dissipation leads to nonequilibrium steady states with a large number of doublon excitations, well above the maximum thermal-equilibrium value. We interpret this effect as an enhancement of local pairing correlations, providing analytical arguments based on a Floquet Hamiltonian. Remarkably, this Hamiltonian shows a tendency to develop long-range staggered superconducting correlations. This suggests the possibility of realizing the elusive $\eta$-pairing phase in driven-dissipative Mott Insulators.
\end{abstract}

DOI: 10.1103/PhysRevB.101.161101

The Floquet engineering of complex quantum systems is a very active line of research in today's condensed matter physics [1]. It consists in the design of periodic perturbations to achieve nonequilibrium driven states remarkably different from their undriven counterparts. Examples are the dynamical control of band topology [2,3] and of magnetic interactions [4] in ultracold atoms in optical lattices, and of effective Hamiltonian parameters in solids under intense laser-pulse excitation [5-7].

A useful description of a periodically driven quantum system is in terms of effective static Hamiltonians derived with large-frequency expansions [8-11]. In general, however, the drive affects also the distribution function of the system, eventually leading to thermalization to a trivial infinitetemperature state $[12,13]$. Nevertheless, when heating can be avoided for finite but long times, interesting prethermal Floquet states can be observed. This is the case, for example, for very large drive frequency [14-17] or systems close to integrability [18-23]. In particular, Ref. [24] showed that strong electronic correlations lead to finite-frequency prethermal states with remarkable properties as a function of drive frequency.

A natural question concerning the Floquet prethermal state is whether the coupling to external reservoirs would cancel out its interesting features, or rather preserve them and possibly make them more accessible. Particularly interesting is the possibility to control the distribution function of the system

\footnotetext{
*fperonaci@pks.mpg.de

†Onleave from Institut de Physique Théorique, Université Paris Saclay, CNRS, CEA, F-91191 Gif-sur-Yvette, France.
}

Published by the American Physical Society under the terms of the Creative Commons Attribution 4.0 International license. Further distribution of this work must maintain attribution to the author(s) and the published article's title, journal citation, and DOI. Open access publication funded by the Max Planck Society. by means of a dissipation mechanism of the energy injected by the drive [25-29].

To investigate this point, in this work we consider the Fermi-Hubbard model with a periodically driven interaction and coupled to a thermal reservoir. Starting from the Mottinsulating phase, our numerical calculations show that the combination of drive and dissipation leads to steady states that are not accessible in the corresponding isolated model. In particular, we reveal a regime with a remarkably large number of high-energy doublon excitations, well above the maximum equilibrium value for the half-filled repulsive Hubbard model.

We interpret this steady-state large double occupancy as an enhancement of local pairing correlations, and we describe the effect as a thermalization to a lowest-order Floquet Hamiltonian. Remarkably, we find that higher-order terms promote finite-momentum doublon superfluidity, namely, staggered long-range pairing correlations among fermions ( $\eta$ pairing), which spontaneously break the hidden $\mathrm{SU}_{\mathrm{C}}(2)$ charge symmetry of the half-filled Hubbard model [30-32]. This suggests a nonequilibrium protocol for Floquet engineering exotic superconducting states in driven-dissipative Mott insulators, as also very recently investigated in similar contexts [33-36].

Our results are relevant for current experiments on laserpumped organic Mott insulators [5,6] and ultracold Fermi gases in driven optical lattices [37,38]. We discuss the latter in particular, suggesting to explore a possibly overlooked regime in future experiments.

Model. The Hamiltonian of the driven-dissipative FermiHubbard model reads $H=H_{\mathrm{Hub}}+H_{\mathrm{diss}}$, where

$$
\begin{aligned}
H_{\mathrm{Hub}} & =\sum_{i j, \sigma} V_{i j} c_{i \sigma}^{\dagger} c_{j \sigma}+U(t) \sum_{i}\left(n_{i \uparrow}-\frac{1}{2}\right)\left(n_{i \downarrow}-\frac{1}{2}\right) \\
H_{\mathrm{diss}} & =\sum_{i \alpha} \omega_{\alpha} b_{i \alpha}^{\dagger} b_{i \alpha}+\lambda \sum_{i \alpha} g_{\alpha}\left(n_{i}-1\right)\left(b_{i \alpha}+b_{i \alpha}^{\dagger}\right)
\end{aligned}
$$




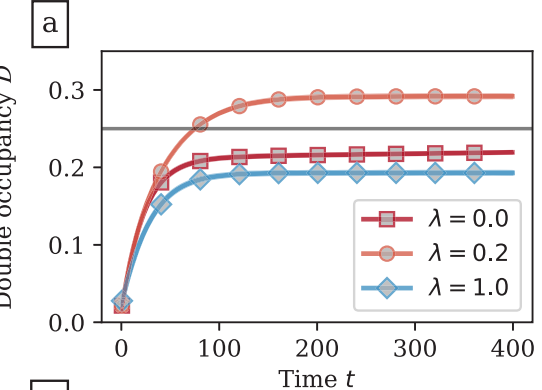

$\mathrm{b}$

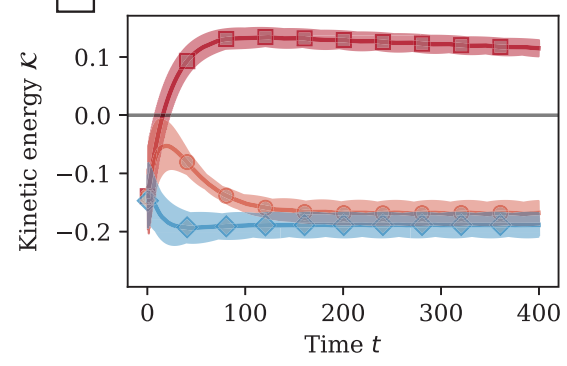

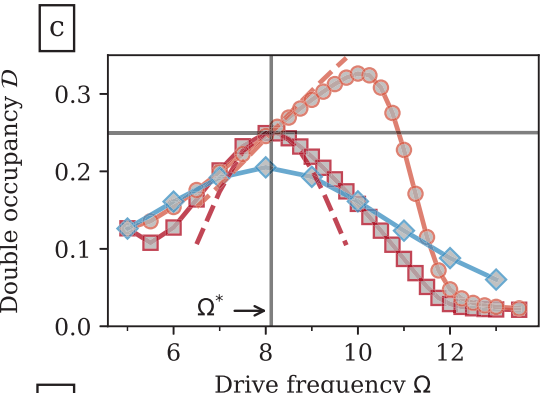

d

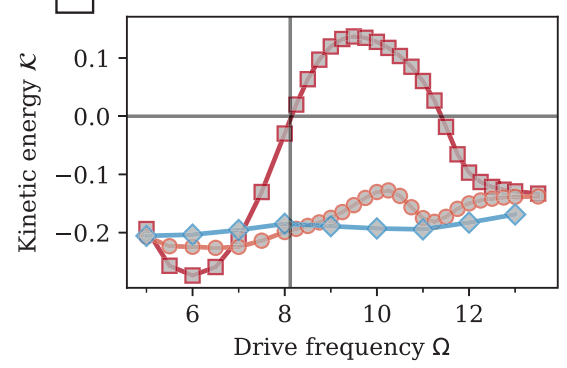

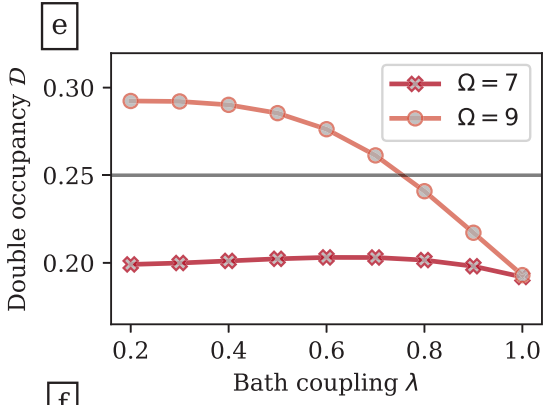

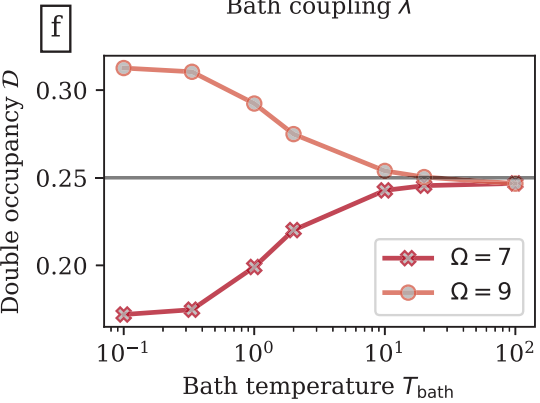

FIG. 1. (a),(b) Time evolution of double occupancy and kinetic energy for drive frequency $\Omega=9>\Omega^{*}$ : oscillations (shaded area) and their average (symbols). Inset in (a) is common to (a)-(d). (c),(d) Long-time averages as a function of drive frequency, and approximate analytical expressions based on Eq. (3) (dashed lines). (e),(f) Long-time average of double occupancy as a function of bath coupling and of bath temperature for $\lambda=0.2$ and drive frequencies $\Omega=7<\Omega^{*}$ and $\Omega=9>\Omega^{*}$.

Here the $c$ 's operators describe fermions hopping with amplitude $V_{i j}$ and subject to a driven local interaction $U(t \geqslant 0)=$ $U_{0}+\delta U \sin \Omega t$. The bare density of states is semicircular with bandwidth $4 V$ and we measure energy, frequency, and inverse of time ( $\hbar=1)$ in units of $V$ [39]. The thermal bath is implemented by independent sets of bosonic modes $b$ which couple to density at each lattice site, with spectral function $J(\omega)=\sum_{\alpha} g_{\alpha}^{2} \delta\left(\omega-\omega_{\alpha}\right) \propto \omega^{2} e^{-\omega / \omega_{c}}\left(\omega_{c}=1\right)$ and coupling $\lambda$ [39]. Importantly, the bath allows energy dissipation but commutes with density $n_{i}=n_{i \uparrow}+n_{i \downarrow}$ and preserves particlehole symmetry. The system remains half filled at all times $\left(\left\langle n_{i \sigma}\right\rangle=0.5\right)$.

Starting from a thermal-equilibrium state, we calculate the time evolution by means of nonequilibrium dynamical mean-field theory (DMFT) [40,41] with the noncrossing approximation as impurity solver $[42,43]$, including the effect of dissipation at weak coupling in $\lambda$ [44] (see Supplemental Material [45] Sec. I for implementation details, and Sec. II for one-crossing-approximation benchmarks). We calculate double occupancy $\mathcal{D}(t)=\left\langle n_{i \uparrow}(t) n_{i \downarrow}(t)\right\rangle$, kinetic energy $\mathcal{K}(t)=$ $\sum V_{i j}\left\langle c_{i \sigma}^{\dagger}(t) c_{j \sigma}(t)\right\rangle$, and local Green's function $G_{\sigma}\left(t, t^{\prime}\right)=$ $-i\left\langle T c_{i \sigma}(t) c_{i \sigma}^{\dagger}\left(t^{\prime}\right)\right\rangle$. For definiteness, we choose $U_{0}=8$ for the initial Mott-insulating state in equilibrium at $T=1$ [46] and $\delta U=2$ for the drive amplitude (see Supplemental Material [45] Sec. VI for a discussion on $\delta U$ ). The bath temperature is $T_{\text {bath }}=1$ unless specified differently. In the absence of dissipation, Floquet prethermalization is observed at all frequencies except for the resonance $\Omega^{*}=8.12 \simeq U_{0}$ [24]. We restrict ourselves to paramagnetic states, leaving the interplay of drive, dissipation, and magnetism to future studies.

Time evolution. In the driven-dissipative model, as well as in the isolated case, double occupancy and kinetic energy display a separation of timescales between fast oscillations synchronized with the drive and a slowly varying average value. However, after a common transient, the thermal reservoir starts to be effective and changes substantially the longtime behavior of both observables.

For weak bath coupling and drive above resonance, the double occupancy grows substantially larger than in the isolated model, going to a stationary average above 0.25 [Fig. 1(a), $\lambda=0.2$ ]. Such a large value would be possible, at equilibrium, only if the interaction were attractive. This striking effect highlights the peculiarity of this nonequilibrium steady state, as we discuss thoroughly below.

Upon increasing the bath coupling [Fig. 1(a), $\lambda=1.0$ ], the double occupancy decreases and eventually remains below the limit of 0.25 at all times. Moreover, we notice that the bath is effective only after a transient time $\sim 1 / \lambda^{2}$, which makes the regime of very weak coupling not accessible by the numerical simulation (see also Ref. [47]).

At the same time, the kinetic energy is also largely affected by dissipation [Fig. 1(b)]. Here the effect is more intuitive: in the isolated model the drive leads to a prethermal state with positive kinetic energy, indicative of a population inversion $[22,24]$. On the other hand, the thermal reservoir dissipates the excess kinetic energy, which remains negative as at equilibrium, and inhibits the population inversion, as we also explicitly show below.

Long-time average. To study the role of drive frequency and bath coupling in a more systematic way, we consider the long-time average of double occupancy and kinetic energy. For weak bath coupling, the dissipative model has double occupancy larger than the isolated one at all frequencies [Fig. 1(c), $\lambda=0.2)]$. However, a remarkable change happens crossing the resonance $\Omega^{*} \simeq U_{0}$. Below resonance, the dissipation has only a quantitative, rather weak effect. In contrast, above resonance, we systematically observe a large increase 
of double occupancy across the limit of 0.25 , as discussed previously for a selected frequency. Lower values are then recovered upon increasing the frequency further, as the system eventually becomes transparent to the drive.

Independent of the bath coupling, the kinetic energy of the dissipative model is rather featureless and negative for all frequencies [Fig. 1(d), $\lambda=0.2,1.0]$. Thus, the thermal reservoir cancels the region of positive kinetic energy characteristic of the isolated case [Fig. 1(d), $\lambda=0.0$ ].

The difference between below and above resonance appears also in the dependence on bath coupling [Fig. 1(e)] and bath temperature [Fig. 1(f)]. Below resonance $(\Omega=7)$ the double occupancy is almost independent of bath coupling and decreases on lowering the bath temperature. Quite differently, above resonance $(\Omega=9)$ it increases on lowering the bath temperature at weak bath coupling.

Notice that the observed behavior does not depend on the details of the bath spectral function as long as we deal with bosonic modes [39]. In contrast, a fermionic reservoir does not lead to the same steady-state large double occupancy (see Supplemental Material [45] Sec. III) because it can change the local density even at zero hopping, spoiling the quasiconservation of doublons which is the basis of Floquet prethermalization in this system [24].

Spectral function. To gain insight into the nature of the steady state, we calculate the spectral function $\bar{A}(\omega)$ and occupation function $\bar{N}(\omega)$ as the average Wigner transforms of the retarded and lesser components of the local Green's function [24]. While the spectral function is the same in the isolated and dissipative models, the occupation function, and thus the distribution function $\bar{F}(\omega)=\bar{N}(\omega) / \bar{A}(\omega)$, changes drastically for drive frequency above resonance and weak bath coupling.

In the isolated model, $\bar{N}(\omega)$ is shifted towards high energy with respect to $\bar{A}(\omega)$ [Fig. 2(a)]. There is therefore a population inversion within the Hubbard bands. Indeed, the local behavior of $\bar{F}(\omega)$ for $\omega \simeq \pm U_{0} / 2$ has the shape of a Fermi function with negative temperature [Fig. 2(c)].

The thermal reservoir completely changes the situation. First, as the dissipation enhances the energy redistribution within the Hubbard bands, $\bar{N}(\omega)$ is pushed back to lower energy [Fig. 2(b)], canceling the population inversion. As a consequence, $\bar{F}(\omega)$ assumes the shape of a Fermi function with positive temperature for $\omega \simeq \pm U_{0} / 2$ [Fig. 2(d)]. Then, the overall weight of $\bar{N}(\omega)$ in the upper band grows and becomes even larger than in the lower band, meaning the creation of a large number of high-energy doublon excitations. These two effects are qualitatively related to the ones discussed above: change of sign of kinetic energy and growth of double occupancy.

Discussion. The above numerical results demonstrate that, in the strongly repulsive Fermi-Hubbard model, the combination of a time-periodic interaction and a dissipative bath leads to steady states with a remarkably large number of doublon excitations. Interestingly, this large double occupancy immediately translates into enhanced local pairing correlations $\mathcal{D}=$ $\left.\left\langle c_{i \uparrow}^{\dagger} c_{i \downarrow}^{\dagger} c_{j \downarrow} c_{j \uparrow}\right\rangle\right|_{i=j}$, although a full calculation of the lattice
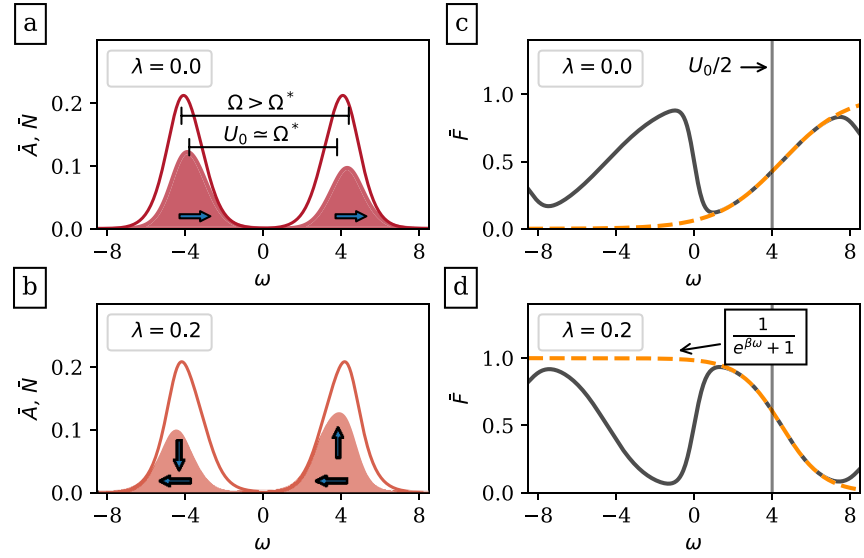

FIG. 2. (a),(b) Long-time average spectral function $\bar{A}(\omega)$ (solid line) and occupation function $\bar{N}(\omega)$ (filled area) of the isolated model $(\lambda=0)$ and the dissipative model $(\lambda=0.2)$ for drive frequency $\Omega=$ $9>\Omega^{*}$. The isolated model (a) has population inversion, signaled by the blueshift of $\bar{N}(\omega)$ with respect to $\bar{A}(\omega)$ (arrows). In the dissipative model (b), the thermal reservoir cancels the population inversion (horizontal arrows) and unveils a nonthermal state with large double occupancy, signaled by the increase of $\bar{N}(\omega)$ in the high-energy band (vertical arrows). (c),(d) Long-time average distribution function $\bar{F}(\omega)$ for the same parameters of (a) and (b) with Fermi-function fit around the Hubbard-band center. The extracted effective temperature is $T_{\text {eff }}=-1.6$ for the isolated model (c) and $T_{\text {eff }}=1.1 \simeq T_{\text {bath }}$ for the dissipative one (d). See Supplemental Material [45] Fig. S3 for a plot of $T_{\text {eff }}(\Omega)$.

susceptibility within DMFT is beyond the scope of this Rapid Communication.

In order to unveil the origin of this effect, let us first consider the isolated model and its Floquet Hamiltonian. To this end, we consider a frequency close to resonance $\Omega \simeq$ $\Omega^{*} \simeq U_{0}$ and perform a rotating-frame transformation on the Hamiltonian (1), followed by a high-frequency expansion [9,48] (see Supplemental Material [45] Sec. V). At lowest order we find

$$
\bar{H}_{\mathrm{Hub}}^{\mathrm{eff}(0)}=V K_{0}+\left(U_{0}-\Omega\right) \sum_{i}\left(n_{i \uparrow}-\frac{1}{2}\right)\left(n_{i \downarrow}-\frac{1}{2}\right) .
$$

Here $K_{0}=\sum\left(V_{i j} / V\right) c_{i \sigma}^{\dagger} c_{j \sigma}\left(n_{i \bar{\sigma}} n_{j \bar{\sigma}}+\bar{n}_{i \bar{\sigma}} \bar{n}_{j \bar{\sigma}}\right)$ are those hopping terms in Eq. (1) that do not alter the number of doubly occupied sites $\left(\bar{n}_{i \sigma}=1-n_{i \sigma}, \bar{\uparrow}=\downarrow\right.$, and $\left.\bar{\downarrow}=\uparrow\right)$. Equation (3) can be interpreted as a Hamiltonian of doublons and holons, where the first term contains hopping and the second term acts as a chemical potential. The numerical results around $\Omega^{*} \simeq U_{0}$ in the isolated model are qualitatively captured in terms of thermalization to this effective Hamiltonian. Indeed, we can extract the effective temperature $T_{\text {eff }}(\Omega) \simeq\left(U_{0}-\Omega\right)^{-1}$ (see Supplemental Material [45] Fig. S3) and, since $\left|T_{\text {eff }}\right| \gg V$ for $\Omega \simeq \Omega^{*}$, we can disregard the kinetic term in Eq. (3) and calculate $\mathcal{D}=$ $0.5\left\{1+\exp \left[0.5\left(U_{0}-\Omega\right) / T_{\text {eff }}\right]\right\}^{-1} \simeq 0.25-\left(\Omega-U_{0}\right)^{2}$. This captures the quadratic behavior for $\lambda=0.0$ [Fig. 1(c), dashed line] with finite-hopping corrections responsible for the quantitative mismatch. 
We now turn to the dissipative model. Here two observations are crucial. First, the enhancement of double occupancy is most pronounced for weak bath coupling [see Fig. 1(e)]. Second, the dissipation leaves largely unchanged the spectral function of the system, while it profoundly changes its occupation (see Fig. 2). On this basis, we argue that at weak coupling the bath does not change the Floquet Hamiltonian, but only affects the effective temperature $T_{\text {eff }} \simeq T_{\text {bath }}=1$ (see Supplemental Material [45] Fig. S3). This leads to $\mathcal{D} \simeq$ $0.25+\left(\Omega-U_{0}\right)$ which qualitatively reproduces the behavior for $\lambda=0.2$ around $\Omega \simeq \Omega^{*}$ [Fig. 1(c), dashed line] where again the mismatch is due to the finite hopping in Eq. (3).

The outcome of this analysis is that, at least for moderately high temperatures $T_{\text {bath }} \simeq V$, the driven-dissipative protocol of Eqs. (1) and (2) leads at long times to thermal states of the doublon Hamiltonian (3). Singly occupied sites, which are relevant in the transient dynamics during doublon-holon proliferation, are not relevant for the steady-state physics, and can be considered as a reservoir of energy and particles to the doublon-holon system.

A natural question now is whether the enhanced local pairing correlations can propagate through the lattice giving a superfluid state of doublons. To answer this, we consider the next order in the Floquet Hamiltonian [45]:

$$
\begin{aligned}
\bar{H}_{\mathrm{Hub}}^{\text {eff(1) }}= & {\left[-i \mathcal{J}_{1}\left(\frac{\delta U}{\Omega}\right) V K_{+}+\text {H.c. }\right] } \\
& +\frac{V^{2}}{\Omega}\left[\mathcal{J}_{0}\left(\frac{\delta U}{\Omega}\right)\right]^{2}\left[K_{+}, K_{-}\right] .
\end{aligned}
$$

Here $\mathcal{J}_{n}(x)$ is the $n$ th-order Bessel function of the first kind, $K_{+}=\sum\left(V_{i j} / V\right) c_{i \sigma}^{\dagger} c_{j \sigma} n_{i \bar{\sigma}} \bar{n}_{j \bar{\sigma}}=\left(K_{-}\right)^{\dagger}$, and one has to note that, in the case of weak drive amplitude considered here, $\mathcal{J}_{n}(\delta U / \Omega) \sim(\delta U / \Omega)^{n}$ and therefore all terms in Eq. (4) indeed vanish as the inverse drive frequency.

The first two terms in parentheses in Eq. (4) create or annihilate doublon excitations, controlling the transient dynamics. However, these processes are largely inhibited in the steady state. Indeed, these terms depend strongly on the drive amplitude $\delta U$, which controls the transient timescale but does not influence the long-time steady state, as found in both the isolated [24] and the dissipative models (see Supplemental Material [45] Sec. V).

The last term in Eq. (4) is similar to the Schrieffer-Wolff result [48,49], which is retrieved for $\delta U \rightarrow 0$ at fixed $\Omega=U_{0}$. It contains several contributions such as density and exchange interactions, and correlated three-site hopping processes. At equilibrium and half filling, in the relevant limit of zero double occupancy, this gives the usual antiferromagnetic Heisenberg model. In far-from-equilibrium situations, if a sizable population of doublons is achieved as in the present case, the same term leads to a completely different physics (see also Ref. [50]).

To discuss Hamiltonian (4) on states with very large double occupancy, it is instructive to neglect processes involving singly occupied sites and rewrite it as [45]

$$
\bar{H}_{\mathrm{Hub}}^{\mathrm{eff}}=J_{\mathrm{eff}} \sum_{\langle i j\rangle}\left(c_{i \uparrow}^{\dagger} c_{i \downarrow}^{\dagger} c_{j \downarrow} c_{j \uparrow}+n_{i \uparrow} n_{i \downarrow} \bar{n}_{j \uparrow} \bar{n}_{j \downarrow}\right) .
$$

Here $J_{\text {eff }}=2 V^{2} / \Omega\left[\mathcal{J}_{0}(\delta U / \Omega)\right]^{2}$, the first term is a doublon hopping, and the second term a first-neighbor doublon interaction. It is now convenient to consider a transformation on spin-down operators $c_{i \downarrow} \rightarrow \tilde{c}_{i \downarrow}=(-1)^{i} c_{i \downarrow}^{\dagger}$ which recasts Eq. (5) as $\bar{H}_{\text {Hub }}^{\text {eff }}=-J_{\text {eff }} \sum \boldsymbol{\eta}_{i} \cdot \boldsymbol{\eta}_{j}$, namely, as an isotropic ferromagnetic Heisenberg model for the so-called $\eta$ spins $\boldsymbol{\eta}_{i}=$ $\frac{1}{2} \sum_{\alpha \beta} \tilde{c}_{i \alpha}^{\dagger} \sigma_{\alpha \beta} \tilde{c}_{i \beta}$ [45]. The invariance under $\eta$-spin rotation is associated to the charge $\mathrm{SU}_{\mathrm{C}}(2)$ symmetry of Hamiltonian (1) which can be used to build eigenstates of the Hubbard model with staggered long-range superconducting correlations $(\eta$ pairing) [30,31]. These same correlations are encoded in Eq. (5). Indeed, the $\eta$-spin ferromagnetic Heisenberg model has magnetization $\left\langle\eta^{z}\right\rangle=\mathcal{D}-0.5$ and below a critical temperature $\sim J_{\text {eff }}$ it has finite order parameter in the $x y$ plane, which corresponds to staggered long-range pairing correlations $\left\langle c_{i \uparrow}^{\dagger} c_{i \downarrow}^{\dagger}+\right.$ H.c. $\rangle=(-1)^{i}\left\langle 2 \eta_{i}^{x}\right\rangle=(-1)^{i} \sqrt{4 \mathcal{D}(1-\mathcal{D})}$.

We stress again that here, for simplicity, we do not consider the interplay between doublons and singly occupied sites, which would result in additional terms in Eq. (5). Moreover, we notice that the $\mathrm{SU}_{\mathrm{C}}(2)$ symmetry implies a degeneracy between the $x y$ plane and the $z$ axis of the $\eta$ spin, which translates into a competition between superfluidity and charge-density wave. We leave the investigation of these issues for future work.

The model system investigated here can be realized in current experimental platforms. Particularly promising are Mott-insulating organic molecular crystals, where laser excitations can induce an effective time-periodic modulation of interaction [5,6]. More direct control is achieved with ultracold atoms in optical lattices. Recent experiments $[37,38]$ have studied the Floquet prethermal state and remarkably found large double occupancy for drive above resonance. We suggest that also in these cases a key role is played by dissipation, which is unavoidable even in cold atoms. Finally, we notice that these experiments have focused on the regime where the effective Hamiltonian reduces to a renormalized Hubbard model. In contrast, here we have studied the case of a doublon-only Floquet Hamiltonian. Therefore, we suggest future experiments to investigate this latter regime to detect the presence of staggered pairing correlations.

Conclusions. In this work, we have studied the combination of a periodically driven interaction and a dissipative bath in the strongly repulsive Fermi-Hubbard model. For weak bath coupling and frequency in a range above the resonance of the isolated model [24], we find a large increase of double occupancy, well above the maximum equilibrium value, which we interpret as an enhancement of local pairing correlations, and understand in terms of thermalization to the lowest-order Floquet Hamiltonian.

Remarkably, the next-order Floquet Hamiltonian contains terms which promote staggered pairing correlations. Therefore, provided a nonequilibrium protocol to reach low enough effective temperatures (see, e.g., Ref. [51]) and eventually further increase the doublon density, the steady state of the driven-dissipative Fermi-Hubbard model would contain off-diagonal staggered long-range order ( $\eta$ pairing), hence a superfluid phase of doublon excitations, similarly to what was very recently found in similar models $[35,36]$. 
Acknowledgments. This work was supported by the European Research Council Grants No. ERC-319286-QMAC (F.P.) and No. ERC-278472-MottMetals (F.P. and O.P.). The Flatiron Institute is a division of the Simons Foundation.
M.S. acknowledges support from a grant "Investissements d'Avenir" from LabEx PALM (Grant No. ANR-10-LABX0039-PALM) and from the CNRS through the PICS-USA14750.
[1] T. Oka and S. Kitamura, Annu. Rev. Condens. Matter Phys. 10, 387 (2018).

[2] T. Oka and H. Aoki, Phys. Rev. B 79, 081406(R) (2009).

[3] G. Jotzu, M. Messer, R. Desbuquois, M. Lebrat, T. Uehlinger, D. Greif, and T. Esslinger, Nature (London) 515, 237 (2014).

[4] F. Görg, M. Messer, K. Sandholzer, G. Jotzu, R. Desbuquois, and T. Esslinger, Nature (London) 553, 481 (2018).

[5] Y. Kawakami, S. Iwai, T. Fukatsu, M. Miura, N. Yoneyama, T. Sasaki, and N. Kobayashi, Phys. Rev. Lett. 103, 066403 (2009).

[6] R. Singla, G. Cotugno, S. Kaiser, M. Först, M. Mitrano, H. Y. Liu, A. Cartella, C. Manzoni, H. Okamoto, T. Hasegawa, S. R. Clark, D. Jaksch, and A. Cavalleri, Phys. Rev. Lett. 115, 187401 (2015).

[7] A. Subedi, A. Cavalleri, and A. Georges, Phys. Rev. B 89, 220301(R) (2014).

[8] N. Goldman and J. Dalibard, Phys. Rev. X 4, 031027 (2014).

[9] M. Bukov, L. D’Alessio, and A. Polkovnikov, Adv. Phys. 64, 139 (2015).

[10] N. Tsuji, T. Oka, P. Werner, and H. Aoki, Phys. Rev. Lett. 106, 236401 (2011).

[11] S. Kitamura and H. Aoki, Phys. Rev. B 94, 174503 (2016).

[12] A. Lazarides, A. Das, and R. Moessner, Phys. Rev. Lett. 112, 150401 (2014).

[13] L. D'Alessio and M. Rigol, Phys. Rev. X 4, 041048 (2014).

[14] D. A. Abanin, W. De Roeck, and F. Huveneers, Phys. Rev. Lett. 115, 256803 (2015).

[15] T. Mori, T. Kuwahara, and K. Saito, Phys. Rev. Lett. 116, 120401 (2016).

[16] T. Kuwahara, T. Mori, and K. Saito, Ann. Phys. 367, 96 (2016).

[17] D. A. Abanin, W. De Roeck, W. W. Ho, and F. Huveneers, Phys. Rev. B 95, 014112 (2017).

[18] M. Bukov, S. Gopalakrishnan, M. Knap, and E. Demler, Phys. Rev. Lett. 115, 205301 (2015).

[19] E. Canovi, M. Kollar, and M. Eckstein, Phys. Rev. E 93, 012130 (2016).

[20] S. A. Weidinger and M. Knap, Sci. Rep. 7, 45382 (2016).

[21] K. Seetharam, P. Titum, M. Kolodrubetz, and G. Refael, Phys. Rev. B 97, 014311 (2018).

[22] A. Herrmann, Y. Murakami, M. Eckstein, and P. Werner, Europhys. Lett. 120, 57001 (2018).

[23] Y. Baum, E. P. L. van Nieuwenburg, and G. Refael, SciPost Phys. 5, 017 (2018).

[24] F. Peronaci, M. Schiró, and O. Parcollet, Phys. Rev. Lett. 120, 197601 (2018).

[25] T. Iadecola, D. Campbell, C. Chamon, C. Y. Hou, R. Jackiw, S. Y. Pi, and S. V. Kusminskiy, Phys. Rev. Lett. 110, 176603 (2013).

[26] T. Iadecola, T. Neupert, and C. Chamon, Phys. Rev. B 91, 235133 (2015).

[27] K. I. Seetharam, C.-E. Bardyn, N. H. Lindner, M. S. Rudner, and G. Refael, Phys. Rev. X 5, 041050 (2015).

[28] K. I. Seetharam, C.-E. Bardyn, N. H. Lindner, M. S. Rudner, and G. Refael, Phys. Rev. B 99, 014307 (2019).
[29] O. Hart, G. Goldstein, C. Chamon, and C. Castelnovo, Phys. Rev. B 100, 060508(R) (2019).

[30] C. N. Yang, Phys. Rev. Lett. 63, 2144 (1989).

[31] S. Zhang, Phys. Rev. Lett. 65, 120 (1990).

[32] D. Mitra, P. T. Brown, E. Guardado-Sanchez, S. S. Kondov, T. Devakul, D. A. Huse, P. Schauß, and W. S. Bakr, Nat. Phys. 14, 173 (2018).

[33] T. Kaneko, T. Shirakawa, S. Sorella, and S. Yunoki, Phys. Rev. Lett. 122, 077002 (2019).

[34] J. Tindall, B. Buča, J. R. Coulthard, and D. Jaksch, Phys. Rev. Lett. 123, 030603 (2019).

[35] P. Werner, J. Li, D. Golež, and M. Eckstein, Phys. Rev. B 100, 155130 (2019).

[36] J. Li, D. Golez, P. Werner, and M. Eckstein, arXiv:1908.08693.

[37] M. Messer, K. Sandholzer, F. Görg, J. Minguzzi, R. Desbuquois, and T. Esslinger, Phys. Rev. Lett. 121, 233603 (2018).

[38] K. Sandholzer, Y. Murakami, F. Görg, J. Minguzzi, M. Messer, R. Desbuquois, M. Eckstein, P. Werner, and T. Esslinger, Phys. Rev. Lett. 123, 193602 (2019).

[39] We expect our results to hold also for other lattices and bath parameters, as the key ingredients-strong local correlations and energy dissipation-do not significantly depend on these details. In Supplemental Material [45] Secs. VII and VIII we provide additional data on hypercubic lattice and with different bath parameters.

[40] A. Georges, G. Kotliar, W. Krauth, and M. J. Rozenberg, Rev. Mod. Phys. 68, 13 (1996).

[41] H. Aoki, N. Tsuji, M. Eckstein, M. Kollar, T. Oka, and P. Werner, Rev. Mod. Phys. 86, 779 (2014).

[42] M. Eckstein and P. Werner, Phys. Rev. B 82, 115115 (2010).

[43] A. Rüegg, E. Gull, G. A. Fiete, and A. J. Millis, Phys. Rev. B 87, 075124 (2013).

[44] H. T. Chen, G. Cohen, A. J. Millis, and D. R. Reichman, Phys. Rev. B 93, 174309 (2016).

[45] See Supplemental Material at http://link.aps.org/supplemental/ 10.1103/PhysRevB.101.161101 for details on the numerical implementation, benchmarks with one-crossing approximation, effective temperature analysis, derivation of the Floquet Hamiltonian, and additional data with fermionic bath and with different drive amplitude, lattice bare density of states, and bath spectral function.

[46] Our results do not qualitatively depend on $U_{0}$ and $T$, as long as the initial state is in the Mott-insulating phase.

[47] M. Eckstein and P. Werner, Phys. Rev. Lett. 110, 126401 (2013).

[48] M. Bukov, M. Kolodrubetz, and A. Polkovnikov, Phys. Rev. Lett. 116, 125301 (2016).

[49] A. H. MacDonald, S. M. Girvin, and D. Yoshioka, Phys. Rev. B 37, 9753 (1988).

[50] A. Rosch, D. Rasch, B. Binz, and M. Vojta, Phys. Rev. Lett. 101, 265301 (2008).

[51] P. Werner, M. Eckstein, M. Müller, and G. Refael, Nat. Commun. 10, 5556 (2019). 